\title{
Expanding the functional role of long noncoding RNAs
}

\author{
Cell Research (2014) 24:1284-1285. doi:10.1038/cr.2014.104; published online 8 August 2014
}

New findings bring to light a previously unappreciated mechanism involved in the regulation of the oncoprotein MYC. Interesting observations find that the long noncoding RNA (IncRNA) PVT1 is active in controlling levels of MYC through regulation of MYC protein stability.

The oncogene MYC is localized in the chromosomal region $8 \mathrm{q} 24.21$, a region frequently amplified in many human tumors. In addition to $M Y C$, this locus also hosts the GSDMC and $C C D C 26$ genes as well as the lncRNA PVT1.A gain of function for all of these genes is observed in many tumors along with $M Y C$.

In the paper by Tseng et al. [1], the authors investigate whether low copy number gain of one, or more, of the genes on $8 \mathrm{q} 24.21$ promotes cancer. In human breast and ovarian cancers, gain of $8 \mathrm{q} 24.21$ is often accompanied by ERBB2/HER2 amplification [2, $3]$. Therefore, by using the $M M T$ Vneu transgenic mice, which harbor amplified Erbb2/Her2, the authors generated a series of mouse models: $\operatorname{gain}(M y c)$, gain (Pvt1,Ccdc26,Gsdmc) and gain (Myc, Pvt1,Ccdc26,Gsdmc). The authors observed that single supernumerary of gain $(M y c)$ and gain (Pvt1,Ccdc26,Gsdmc) was not sufficient to promote cancer in the MMTVneu mouse model. In contrast, gain (Myc,Pvt1,Ccdc26, Gsdmc) showed shorter mammary tumor latency and increased penetrance compared to the other genotypes, suggesting that other genetic elements, not only $M y c$, within the 8q24.21 region, are involved in cancer progression. The authors excluded $G s d m c$ and $C c d c 26$ from this process and focused on the functional role of the lncRNA PVT1.

To elaborate their findings, the studies were expanded to human breast cancer cell lines, which harbor high copy gains of 8q24.21. Transcriptional activity of the two genes did not appear linked since knockdown of either $P V T 1$ or $M Y C$ did not affect the mRNA expression of one another. However, cellular proliferation was decreased upon knockdown of $M Y C$ as well as $P V T 1$ and knockdown of both $M Y C$ and $P V T 1$ did not further reduce cellular proliferation, thus suggesting that $M Y C$ and PVTI may act in the same pathway.

Although changes in expression of PVTl had no effect on MYC mRNA expression, it was noted that high levels of $P V T 1$ correlated with increased levels MYC protein and this effect appeared to be a consequence of increased stability of the MYC protein. It is well established that MYC protein degradation is promoted by phosphorylation of threonine 58 (Thr58) (Figure 1A) [4]. The authors speculated and observed that PVT1 protects the MYC protein from phosphorylation-mediated degradation (Figure 1B).

In order to further dissect the mechanism of action, Tseng et al. [1] showed that MYC and PVT1 co-localize in the nucleus. Moreover, RNA immunoprecipitation of MYC supported the notion that MYC and PVT1 are indeed part of the same complex. In addition, the authors applied the CRISPR method to the MYC-driven colon cancer cell line HCT116 and generated PVT1-null cells ( $\triangle$ PVT1-HCT116). In support of their findings, the $\triangle \mathrm{PVT} 1-\mathrm{HCT} 116$ cells
A

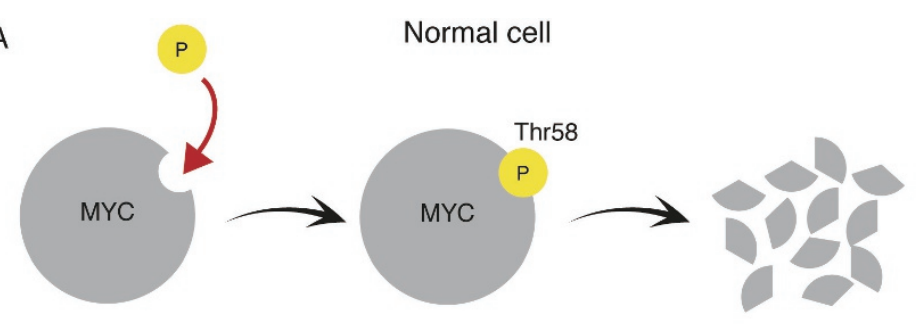

B

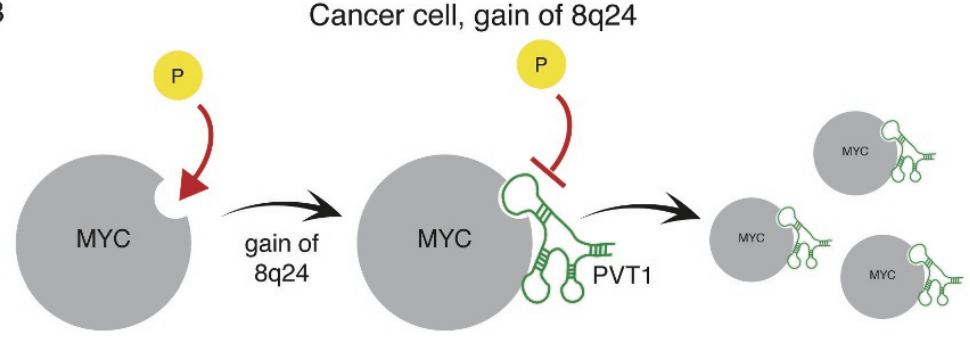

Figure 1 (A) In normal cells, MYC is targeted by phosphorylation on threonine 58 (Thr58) and becomes destabilized and degraded. (B) In cancer cells, gain of 8q24 promotes the expression of MYC and PVT1. PVT1 interferes with the phosphorylation of Thr58 on MYC, which stabilizes the protein and increases its level. 
demonstrated reduced proliferation and impaired colony formation in soft agar, and xenograft studies showed either failed tumor formation or reduced volume compared to the parental HCT116 cells. Finally, using publically available databases, the authors found that $>97 \%$ of tumors with increased 8q24 copy number had increased copy number of both $M Y C$ and PVT1 genes.

In summary, this exciting study provides convincing data that PVT1 is an important regulatory lncRNA, which is involved in modulating the phosphorylation of MYC and cancer progression. The findings presented within this study are in line with previous reports suggesting that phosphorylation of STAT3 is regulated by a lncRNA, lnc-DC [5].

While the functional investigation of lncRNAs has long focused on chromatin remodeling [6], a role beyond chromatin is now emerging. The intriguing studies carried out by Tseng et al. [1] suggest that several questions remain to be ad- dressed. It would indeed be of great interest to precisely investigate how PVTI is interfering with the phosphorylation of MYC. For instance, 1) Is there a specific domain of $P V T 1$, which is responsible for the action [7]? 2) Does PVT1 form direct interactions with MYC thereby blocking the phosphorylation of Thr58? And 3) Does PVT1 interact with other proteins except MYC? By addressing these questions in future studies, it could be possible to identify regulatory sequences within PVT1. This would not only allow for better understanding of the interplay between PVT1 and MYC, but could also lead to the identification of conserved regions and RNA structures. Such structures might also be present in other lncRNAs with similar functions and serve as the basis of potentially new therapeutics to disrupt such interactions and affect cancer progression.

\section{Per Johnsson ${ }^{1}$, Kevin V Morris ${ }^{2,3}$}

${ }^{1}$ Department of Oncology and Pathology, Karolinska Institutet, SE-171 77 Stockholm, Sweden; ${ }^{2}$ Biotechnology and Biomedical Sciences, The University of New South Wales, NSW 2052, Sydney, Australia; ${ }^{3}$ Department of Molecular and Experimental Medicine, The Scripps Research Institute, La Jolla, CA 92037, USA

Correspondence: Kevin V Morris

E-mail: kmorris@unsw.edu.au

\section{References}

1 Tseng YY, Moriarity BS, Gong W, et al. Nature $2014 ; \mathbf{5 1 2}: 82-86$.

2 Park K, Kwak K, Kim J, et al. Hum Pathol 2005; 36:634-639.

3 Al-Kuraya K, Schraml P, Torhorst J, et al. Cancer Res 2004; 64:8534-8540.

4 Yeh E, Cunningham M, Arnold H, et al. Nat Cell Biol 2004; 6:308-318.

5 Wang P, Xue Y, Han Y, et al. Science 2014; 344:310-313.

6 Johnsson P, Ackley A, Vidarsdottir L, et al. Nat Struct Mol Biol 2013; 20:440-446.

7 Johnsson P, Lipovich L, Grandér D, et al. Biochim Biophys Acta 2014; 1840:10631071. 